\title{
Component assembling model and its application to quasi-brittle damage
}

\author{
S.C. Deng *, J.X. Liu, J. Zhang, N.G. Liang \\ State Key Laboratory of Nonlinear Mechanics (LNM), Institute of Mechanics, Chinese Academy of Sciences (CAS), \\ Graduate School of CAS, Beijing 100080, China
}

Available online 25 October 2006

\begin{abstract}
Potential energy can be approximated by "pair-functional" potentials which is composed of pair potentials and embedding energy. Pair potentials are grouped according to discrete directions of atomic bonds such that each group is represented by an orientational component. Meanwhile, another kind of component, the volumetric one is derived from embedding energy. Damage and fracture are the changing and breaking of atomic bonds at the most fundamental level and have been reflected by the changing of these components' properties. Therefore, material is treated as a component assembly, and its constitutive equations are formed by means of assembling these two kinds of components' response functions. This material model is referred to as the component assembling model. Theoretical analysis and numerical computing indicate that the proposed model has the capacity of reproducing some results satisfactorily, with the advantages of physical explicitness and intrinsic induced anisotropy, etc.
\end{abstract}

(C) 2006 Elsevier Ltd. All rights reserved.

Keywords: Damage; Fracture; Anisotropy; Constitutive equation; Pair-functional potentials; Pair potentials; Embedding energy; Orientational/volumetric component; Component assembling model

\section{Introduction}

Material made up of a large number of atoms is regarded as a many-body system. The binding forces among atoms determine the material structures and its intrinsic mechanical and electromagnetic properties [1-3]. The exact treatment of this many-body problem by formulation and solution of Schrodinger wave equations $[1,4]$ is not tractable because of the large number of atoms involved. By using the adiabatic Born-Oppenheimer approximation $[1,5]$, the interatomic potentials that express directly the interactions among atoms have been proposed. The difficulties in solving many-body system have been reduced greatly. Pair-functional is a representative model of interatomic potentials. It considers the interaction among the atoms and electrons [1,3,6-8]. However, the space and time scales in molecular dynamics simulation [9-11] based on interatomic potentials have been confined for the limited computational power.

\footnotetext{
* Corresponding author. Tel.: +86106254 5533 3182; fax: +86 1062579511.

E-mail address: dengsc@lnm.imech.ac.cn (S.C. Deng).
} 
From the viewpoint of continuum mechanics [12,13], material deforms under external actions, and its mechanical property is directly derived as soon as the rules of energy changing during deformation are known. However, it is difficult to get these rules in such complex situations. The properties of material are not unalterably determined by their average chemical composition but they are to a large extent influenced by their microstructures. The microstructures of the material change under external actions. With loads continually increasing, microdefects such as microcracks and microvoids begin to nucleate and grow, and material mechanical properties degrade accordingly. The process of material degrading has been explored by damage mechanics [14-18]. The damage constitutive relation is difficult to derive and the corresponding formulation can be complex, if anisotropic damage and dissipative potentials and internal variables are considered.

Considering the difficulties and merits in continuum mechanics and interatomic potentials, many researchers began to set up macroscopic constitutive models directly from interatomic potentials or combine these two theories, e.g. the quasi-continuum (QC) model [19,20], the virtual internal bond (VIB) model [21] and the relations between molecular dynamics and micromorphic theories [22,23].

In this paper, the component assembling model has been proposed and extended to damage. Potential energy due to deformations has been expressed in the form of the sum of pair-functional potentials. In which, pair potentials has been computed according to discrete directions which are determined by the interactions among atoms, and the corresponding orientational component has been established, i.e., the sum of pair potentials parallel to the specified direction is the energy of the corresponding orientational component and the stiffness contribution of this part of atomic bonds is its stiffness. As a typical 1-D component, the orientational one bears tensile or compressive loadings. Meanwhile, the other kind of component - the volumetric one has also been proposed from embedding energy. For the density of electronic gas only relating to volume, the volumetric component is a typical 3-D one and it can bear hydrostatic stress. As macroscopic phenomena of the changing and breaking of atomic bonds, damage and fracture have been reflected by the changing of stiffness and state of these components. Particularly, anisotropy has been expressed naturally by the concept of components. The constitutive equations considering damage have been formulated by assembling these two kinds of components' response functions.

The remainder of this paper is organized as follows. In part 2, the proposed model named component assembling one will be derived elaborately. In parts 3, the proposed model of homogeneous and isotropic materials will be introduced respectively. Quasi-brittle damage is considered in part 4 and the corresponding numerical simulation will be done in part 5. In the last part, a concise conclusion about the proposed model will be given.

\section{Derivation of component assembling model}

\subsection{Basic kinematic relations}

Let $\varepsilon_{i j}$ denote the strain, and the strain along the vector $\mathbf{r}$ is expressed in the form

$$
\lambda=\varepsilon_{i j} n_{i} n_{j} .
$$

Herein, $\mathbf{n}$ denotes the directional unit vector of $\mathbf{r}$ and $n_{i}$ its $i$ th component. $r$ denotes the length of $\mathbf{r}, r=|r|, r_{i}$ the $i$ th component of $\mathbf{r}$. Thus,

$$
\delta r=r \lambda=\frac{r_{i} r_{j}}{r} \varepsilon_{i j}
$$

The density of electronic gas $\rho$ relates to volume ( $\rho$ decreases as volume expands). For infinitesimal strain, there is

$$
\delta \rho=-\varepsilon_{i j} \delta_{i j} \rho,
$$

where $\delta_{i j}$ denotes the second order identity tensor. Differentiating Eq. (2), it follows that:

$$
\delta^{2} r=\delta(\delta r)=\left(n_{i} n_{j} n_{k} n_{l} \varepsilon_{i j} \varepsilon_{k l}\right) r .
$$

Similarly, there is

$$
\delta^{2} \rho=\varepsilon_{i j} \varepsilon_{k l} \delta_{i j} \delta_{k l} \rho .
$$




\subsection{Derivation of component assembling model}

The principal view of pair-functional is that the cohesive energy of an atom is determined by the local electron density at the site into which that atom is placed $[1-3,6-8]$,

$$
E_{\text {embedding }}=F(\rho) \text {, }
$$

where $F(\rho)$ is referred to as the embedding energy (function), modeling the attractive interaction as a function of the local electron density $\rho$ into which the considered atom is placed. Here, the electron density is given by

$$
\rho_{i}=\sum_{j}^{j \neq i} f\left(R_{i j}\right)
$$

This function can be interpreted as the charge at the $i$ th nucleus due to the spherical symmetric electronic charge densities $f(R)$ of the neighboring atoms. Hence, the function $f(R)$ evaluated at the distance $R_{i j}=\left|\mathbf{R}_{i}-\mathbf{R}_{j}\right|=R^{(i, j)},(i \neq j)$ tells us exactly how much electronic density bleeds off from site $\mathrm{j}$ onto its neighbors. Where $\mathbf{R}_{i}$ denotes the position of the $i$ th nuclear. The embedded atom method [6-8] posits a total energy of the form,

$$
E_{\text {exact }}\left(\left\{\mathbf{R}_{i}, \mathbf{r}_{n}\right\}\right) \rightarrow E_{\text {approx }}\left[\rho(\mathbf{r}),\left\{\mathbf{R}_{i}\right\}\right]=\frac{1}{2} \sum_{i j}^{i \neq j} \phi\left(\mathbf{R}_{i j}\right)+\sum_{i} F\left(\rho_{i}\right),
$$

herein, the term $\phi\left(R_{i j}\right)$ describes a pairwise isotropic interatomic potential function which is essentially repulsive and depends only on the atomic spacing $R_{i j}, r_{n}$ denotes the position of the $n$th electron. The above equation may include various parameters and these parameters can be obtained by fitting equation to intrinsic material parameters such as the elastic constants, crystal structure and cohesive energy, etc.

It is convenient to define zero of energy at initial equilibrium positions. Expanded Eq. (8) by Taylor series, further progress can be made in trimming down the first term $\frac{\partial E_{\text {tot }}}{\partial \mathbf{R}} \cdot \delta \mathbf{R}$ by recognizing that the expansion is built around the equilibrium configuration, and the first term can be eliminated since at equilibrium $\frac{\partial E_{\text {tot }}}{\partial \mathbf{R}} \cdot \delta \mathbf{R} \equiv 0$. Thus, potential energy due to deformation (strain energy) is expressed in the form (the third and higher derivatives excluded)

$$
U=\frac{1}{4} \sum_{(\alpha, \beta)}^{\alpha \neq \beta} \phi^{\prime \prime}\left(R^{(\alpha, \beta)}\right)\left[\delta R^{(\alpha, \beta)}\right]^{2}+\frac{1}{4} \sum_{(\alpha, \beta)}^{\alpha \neq \beta} \phi^{\prime}\left(R^{(\alpha, \beta)}\right) \delta^{2} R^{(\alpha, \beta)}+\frac{1}{2} \sum_{\alpha} F^{\prime \prime}\left(\rho^{(\alpha)}\right)\left[\delta \rho^{(\alpha)}\right]^{2}+\frac{1}{2} \sum_{\alpha} F^{\prime}\left(\rho^{(\alpha)}\right) \delta^{2} \rho^{(\alpha)} .
$$

When computing for strain energy it should be keep in mind that one is that it needs to run over all pairs of interactive atoms for pair potentials and the other is that it should run over all atoms for embedding potentials. While computing the total of pair potentials, the number of pair potentials is larger than the number of atoms in the selected material element, yet the number of directions of interatomic bonds is smaller than the number of atoms due to periodic crystal configurations. Therefore, pair potentials are grouped according to directions, microstructures and their evolutions are embodied on energy changing in different directions. This is the idea of component assembling model. To an appointed unit direction $\mathbf{n}$, an orientational component is set up accordingly. $\mathbf{n}$ denotes the direction of this component and the sum of pair potentials parallel to $\mathbf{n}$ in material is the energy of this orientational one,

$$
E^{(\mathbf{n})}=\frac{1}{4} \sum_{(\alpha, \beta)}^{\mathbf{R}^{(\alpha, \beta)} \| \mathbf{n}}\left\{\phi^{\prime \prime}\left(R^{(\alpha, \beta)}\right)\left[\delta R^{(\alpha, \beta)}\right]^{2}+\phi^{\prime}\left(R^{(\alpha, \beta)}\right) \delta^{2} R^{(\alpha, \beta)}\right\}
$$

Run over all directions of interactive atoms and set up orientational components accordingly. Then, the change of pair potentials $U_{p}$ due to deformation is denoted by these orientational components,

$$
U_{p}=\frac{1}{4} \sum_{(\alpha, \beta)}^{\alpha \neq \beta}\left\{\phi^{\prime \prime}\left(R^{(\alpha, \beta)}\right)\left[\delta R^{(\alpha, \beta)}\right]^{2}+\phi^{\prime}\left(R^{(\alpha, \beta)}\right) \delta^{2} R^{(\alpha, \beta)}\right\}=\sum_{\mathbf{n}} E^{(\mathbf{n})} .
$$


Therefore, strain energy (density) is written as follows:

$$
\begin{aligned}
U & =U_{p}+\frac{1}{2} \sum_{\alpha} F^{\prime \prime}\left(\rho^{(\alpha)}\right)\left[\delta \rho^{(\alpha)}\right]^{2}+\frac{1}{2} \sum_{\alpha} F^{\prime}\left(\rho^{(\alpha)}\right) \delta^{2} \rho^{(\alpha)} \\
& =\sum_{\mathbf{n}} E^{(\mathbf{n})}+\frac{1}{2} \sum_{\alpha} F^{\prime \prime}\left(\rho^{(\alpha)}\right)\left[\delta \rho^{(\alpha)}\right]^{2}+\frac{1}{2} \sum_{\alpha} F^{\prime}\left(\rho^{(\alpha)}\right) \delta^{2} \rho^{(\alpha)} .
\end{aligned}
$$

Considering material element that contains large numbers of grains whose lattice orientations are uniformly distributed, the properties of the aggregate would exhibit little if any anisotropy. In other words, in large enough material element, the directions of pair potentials spread all over the whole space, the number of orientational components tends to infinity, and these components have same characteristic. Eq. (12) has to be re-expressed in integral form, which will be discussed later. The energy density of the orientational component due to deformation is "the strain energy density of the orientational one", and its modulus is deduced conveniently from this kind of strain energy.

Cauchy-Born hypothesis [1,3] builds a bridge between microscopic movements and macroscopic deformations. It asserts that the lattice has been subjected to homogeneous deformation locally. Notes

$$
H^{(\mathbf{n})}=\frac{1}{2} \sum_{(\alpha, \beta)}^{\mathbf{R}^{(\alpha, \beta)} \| \mathbf{n}}\left\{\phi^{\prime \prime}\left(R^{(\alpha, \beta)}\right)\left[R^{(\alpha, \beta)}\right]^{2}+\phi^{\prime}\left(R^{(\alpha, \beta)}\right) R^{(\alpha, \beta)}\right\} .
$$

Substitution of Eq. (13) into Eq. (12), and thinking Cauchy-Born hypothesis, yields the expression

$$
U=\left\{\frac{1}{2} \sum_{\mathbf{n}} H^{(\mathbf{n})} n_{i} n_{j} n_{k} n_{l}+\left[\frac{1}{2} \sum_{\alpha} F^{\prime \prime}\left(\rho^{(\alpha)}\right)\left(\rho^{(\alpha)}\right)^{2}+\frac{1}{2} \sum_{\alpha} F^{\prime}\left(\rho^{(\alpha)}\right) \rho^{(\alpha)}\right] \delta_{i j} \delta_{k l}\right\} \varepsilon_{i j} \varepsilon_{k l} .
$$

By energy-equivalence and comparing with continuum mechanics [12,13], it is found that

$$
C_{i j k l}=\sum_{\mathbf{n}} H^{(\mathbf{n})} n_{i} n_{j} n_{k} n_{l}+\left[\sum_{\alpha} F^{\prime \prime}\left(\rho^{(\alpha)}\right)\left(\rho^{(\alpha)}\right)^{2}+\sum_{\alpha} F^{\prime}\left(\rho^{(\alpha)}\right) \rho^{(\alpha)}\right] \delta_{i j} \delta_{k l} .
$$

The first item on the right of Eq. (15) is derived from pair potentials, and the last two are from embedding energy. In fact, $H^{(\mathbf{n})}$ is the elastic modulus of the orientational component parallel to $\mathbf{n}$. Similarly, the next kind of component - the volumetric one is introduced to represent the contribution of embedding energy, and its bulk modulus is given by

$$
G=\sum_{\alpha} F^{\prime \prime}\left(\rho^{(\alpha)}\right)\left(\rho^{(\alpha)}\right)^{2}+\sum_{\alpha} F^{\prime}\left(\rho^{(\alpha)}\right) \rho^{(\alpha)} .
$$

Therefore, the elasticity tensor is rewritten by pair-functional potentials. Eq. (15) is the constitutive equation of component assembling model, and it can be rewritten as

$$
C_{i j k l}=\sum_{\mathbf{n}} H^{(\mathbf{n})} n_{i} n_{j} n_{k} n_{l}+G \delta_{i j} \delta_{k l} .
$$

The elasticity tensor satisfies the Voigt symmetry: $C_{i j k l}=C_{j i k l}=C_{i j l k}=C_{k l i j}$. In addition, due to introducing the volumetric component, it overcomes the constraint of the Cauchy relation, $C_{i j k l}=C_{i k j l}$.

\section{Component assembling model of homogeneous and isotropic materials}

Once the formula of pair-functional potentials is specified and the lattice configuration is known, the crystal properties can be derived directly. Actually, all materials are inhomogeneous and anisotropic, but lots of materials demonstrate macroscopic isotropy, such as most metals. In particular, for homogeneous material (the observing length is much larger than the characteristic length), the directions between nucleus spread allover the space, and the electron density can be treated as a constant, that is, it admits the representation 


$$
H(\theta, \varphi) \Delta \Omega=H(\theta, \varphi) \sin \varphi \Delta \theta \Delta \varphi=\frac{1}{2} \sum_{(\alpha, \beta)}^{\mathbf{R}^{(\alpha, \beta)} \in \Delta \Omega}\left\{\phi^{\prime \prime}\left(R^{(\alpha, \beta)}\right)\left(R^{(\alpha, \beta)}\right)^{2}+\phi^{\prime}\left(R^{(\alpha, \beta)}\right) R^{(\alpha, \beta)}\right\},
$$

herein $\Delta \Omega$ is a little solid angle around the direction $(\theta, \varphi)$, and

$$
G(\theta, \varphi)=\left[\sum_{\alpha} F^{\prime \prime}\left(\rho^{(\alpha)}\right)\left(\rho^{(\alpha)}\right)^{2}+\sum_{\alpha} F^{\prime}\left(\rho^{(\alpha)}\right) \rho^{(\alpha)}\right] .
$$

It is the bulk modulus of the volumetric component. Because the changes of electronic density do only relate to volumetric strain, and infinitesimal deformation is considered, Eq. $\left(16^{\prime}\right)$ becomes

$$
G(\theta, \varphi) \equiv G
$$

The summation form in Eq. (15) is rewritten in integral form

$$
C_{i j k l}=\int_{0}^{\frac{\pi}{2}} \int_{0}^{2 \pi} H(\theta, \varphi) n_{i}(\theta, \varphi) n_{j}(\theta, \varphi) n_{k}(\theta, \varphi) n_{l}(\theta, \varphi) \sin \theta \mathrm{d} \theta \mathrm{d} \varphi+G \delta_{i j} \delta_{k l} .
$$

The above equation is the constitutive equation for homogeneous materials.

The integral of Eq. (18) can be transformed to discrete summation for numerical computing, and the selected directions turned into the orientational components' directions. Concretely, the selected representative directions on the space as the directions of orientational components, the potential energy parallel to the directions in a certain angle range is computed as the potential energy of the orientational component, and this part of energy contribution to the stiffness modulus is transformed to the stiffness modulus of this component,

$$
k^{(\theta, \phi)}=H(\theta, \phi) \Delta \mathbf{\Omega}^{(\theta, \phi)}=H(\theta, \phi) \sin \phi \Delta \theta \Delta \phi .
$$

Material elasticity tensor is expressed by these two kinds of components

$$
C_{i j k l}=\sum_{(\theta, \phi)} k^{(\theta, \phi)} n_{i}^{(\theta, \phi)} n_{j}^{(\theta, \phi)} n_{k}^{(\theta, \phi)} n_{l}^{(\theta, \phi)}+G \delta_{i j} \delta_{k l}=\sum_{s} k^{s} n_{i}^{s} n_{j}^{s} n_{k}^{s} n_{l}^{s}+G \delta_{i j} \delta_{k l}
$$

or

$$
\mathbf{C}=\sum_{s} k^{s} \mathbf{n}^{s} \otimes \mathbf{n}^{s} \otimes \mathbf{n}^{s} \otimes \mathbf{n}^{s}+G \mathbf{I}_{2} \otimes \mathbf{I}_{2}
$$

herein $\mathbf{C}$ denotes the elasticity tensor, $k^{s}$ and $\mathbf{n}^{s}$ the initial intact modulus and unit direction of the $s^{\text {th }}$ orientational component respectively. $\mathbf{I}_{2}$ denotes the second-order identity. Anisotropy is embodied intrinsically on

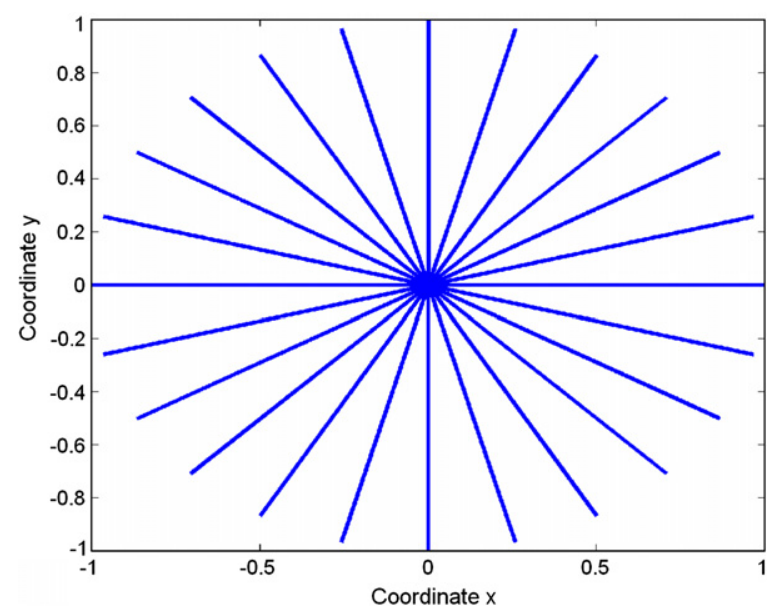

Fig. 1. Configuration of planar discrete orientational components. 


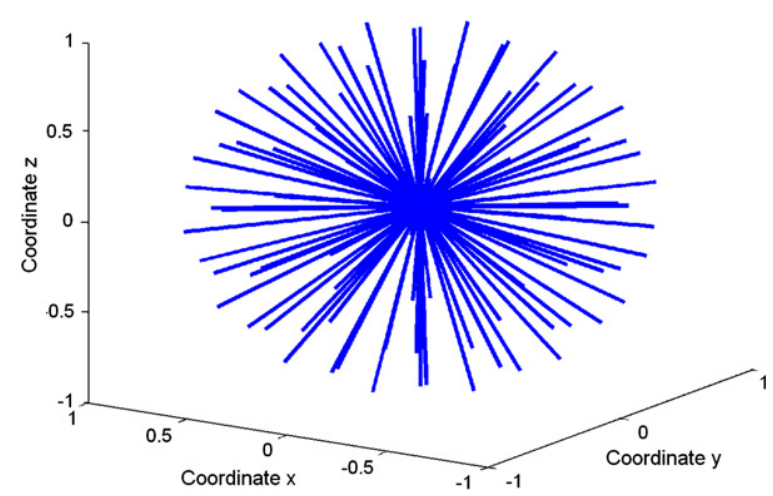

Fig. 2. Configuration of spatial discrete orientational components.

the different moduli of different orientational components. Eq. (19) or Eq. (19) is the constitutive equation of the proposed model. Figs. 1 and 2 are the configurations of planar and spatial discrete orientational components respectively (12 components in Fig. 1 and 46 ones in Fig. 2).

In particular, for homogeneous and isotropic materials, there stands

$$
H(\theta, \varphi) \equiv H .
$$

Integrating Eq. (18) on the up half a unit sphere, and yields,

$$
C_{i j k l}=\left(\frac{2 \pi}{15} H+G\right) \delta_{i j} \delta_{k l}+\frac{2 \pi}{15} H\left(\delta_{i k} \delta_{j l}+\delta_{i l} \delta_{j k}\right) .
$$

Comparing with traditional continuum mechanics, yields the result

$$
\left\{\begin{array}{l}
H=\frac{15}{2 \pi} \mu, \\
G=\lambda-\mu,
\end{array}\right.
$$

where $\lambda$ and $\mu$ denote Lame's coefficients.

\section{Quasi-brittle damage}

Fracture of engineering structures is often preceded by considerable changes in the microstructures of the material they are made of. Examples are microcracking in concrete, fibre pull-out or delamination in composites and the formation of voids in ductile metals. Accurate failure predictions can only be obtained if this microstructural damage is taken into account in the fracture modeling. This requirement has led to the development of so-called local or continuum approaches to fracture, in which fracture is regarded as the ultimate consequence of the material degradation process [24,25]. In these methods, the degradation is often modeled using continuum damage mechanics [14,15,17,18,25-27]. Continuum damage theories introduce a set of field variables (damage variables) which explicitly describe the local loss of material integrity. A crack is represented by that part of the material domain in which the damage has become critical, i.e. where the material cannot sustain stress anymore. Redistribution of stresses results in the concentration of deformation and damage growth in a relatively small region in front of crack tip. It is the growth of damage in this process zone which determines in which direction and at which rate the crack will propagate. Crack initiation and growth thus follow naturally from the standard continuum theory, instead of from separate fracture criteria.

Microstructure change and damage by fracture are related to the breaking of atomic bonds. The orientational component is an abstract of atomic bonds. When atomic debonding emerges, the corresponding orientational component changes their mechanical properties e.g. stiffness. The more atomic debonding occurs and the more stiffness changes,

$$
D=1.0-\frac{k^{\prime}}{k}
$$


where $k$ and $k^{\prime}$ denote the initial and instantaneous(damaged) secant stiffness of the component respectively, $D$ its damage factor. A scalar value is enough for a 1-D component, it is a microscopic value (however, for material element, its constitutive is a typical fourth order tensor, as the following Eq. (27)). Meantime, as a typical 1-D component, the orientational one has simple constitutive relation, it is expressed as follows:

$$
f=(1-D) k \lambda,
$$

herein $f$ denotes the stress of the component and $\lambda$ its strain. Take $\mathbf{n}$ as the direction of the component. There is $\lambda=\varepsilon_{i j} n_{i} n_{j}$, and Eq. (22) becomes

$$
f=(1-D) k \varepsilon_{i j} n_{i} n_{j}
$$

and its rate form is

$$
\dot{f}=(1-D) k n_{i} n_{j} \dot{\varepsilon}_{i j}-k n_{i} n_{j} \varepsilon_{i j} \dot{D} .
$$

The stress contribution of single orientational component is rewritten as

$$
\sigma_{i j}=f n_{i} n_{j} .
$$

For simplicity, the stiffness $G$ can be regarded as a constant during deformation. Meanwhile, the hypothesis that damage is never healed up has been adopted,

$$
0 \leqslant D \leqslant 1, \quad \dot{D} \geqslant 0 \text {. }
$$

The damage of the orientational component has been treated as a function of deformation history $\lambda_{h}$ (the maximum/minimum strain in whole deformation process):

$$
D=D\left(\lambda_{h}\right) \text {. }
$$

Consider the notation

$$
\frac{\mathrm{d} D}{\mathrm{~d} \lambda_{h}}= \begin{cases}\frac{\mathrm{d} D}{\mathrm{~d} \lambda_{h}}, & \text { when } \lambda=\lambda_{h} \text { and } \lambda \dot{\lambda}>0 \\ 0, & \text { other conditions. }\end{cases}
$$

It follows that:

$$
\dot{D}=\frac{\mathrm{d} D}{\mathrm{~d} \lambda_{h}} \dot{\lambda}
$$

Substitution of Eqs. (26) and (22) into Eq. (23) and considering the volumetric component, yields the material damage constitutive equations

$$
\dot{\sigma}_{i j}=\left\{\sum_{s=1}^{N}\left\{\left[\left(1-D^{s}\right) k^{s}-k^{s} \lambda^{s}\left(\frac{\mathrm{d} D}{\mathrm{~d} \lambda_{h}}\right)^{s}\right] n_{i}^{s} n_{j}^{s} n_{k}^{s} n_{l}^{s}\right\}+G \delta_{i j} \delta_{k l}\right\} \dot{\varepsilon}_{k l}=C_{i j k l} \dot{\varepsilon}_{k l} .
$$

Compared with other anisotropic damage models [15-18,25-27], the proposed one is also anisotropic. In addition, its elasto-damage stiffness is intrinsic symmetry and the artificially symmetrical treatments in traditional anisotropic damage models are no longer needed. The evolutions of these components are very important in practice. In general, these evolutions can be obtained by fitting experiment data, the methods about it will be discussed extensively in the future.

\section{Numerical example}

In this part, the capacities of the present model will be analyzed preliminarily by an example.

In this example, the field will be integrated numerically by $2 \times 2$ points Gauss integration, and quadrilateral element with 4 nodes is adopted. A thin plate of the length $L=100 \mathrm{~mm}$ and the width $d=10 \mathrm{~mm}$ will be analyzed under tensional loading, the thickness of the plate $t=1 \mathrm{~mm}$. For simplicity, a plane stress situation is assumed. The planar discretization of orientational components illustrates as Fig. 1. Young's modulus $E=20,000 \mathrm{~N} / \mathrm{mm}^{2}$ and Poisson's ratio $v=1 / 3$, see Fig. 3. In order to trigger localization, the Young's mod- 


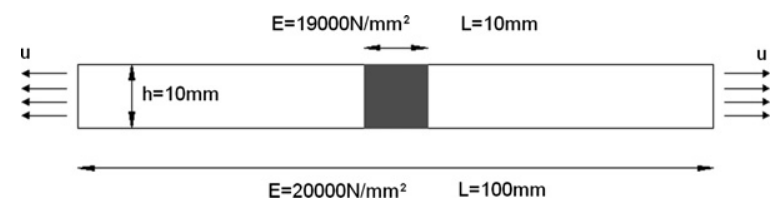

Fig. 3. Two-dimensional plate with imperfection-geometry.

ulus has been reduced by $5 \%$ in a $l=10 \mathrm{~mm}$ wide zone in the middle of the plate, see also Fig. 3 . The system is modeled by $20 \times 2,20 \times 4$ and $40 \times 4$ elements, respectively. For simplicity, the stress-strain curve of orientational components is illustrated by Fig. 4, and Fig. 5 is the corresponding damage-strain curve. The volumetric component is treated as a constant. The load-deflecting curve is shown in Fig. 6, and the evolution of longitudinal strain distribution in plate with imperfection is illustrated in Fig. $7(\mathrm{a}, 20 \times 2$; b, 20 $\times 4$; c, $40 \times 4$ ). From which, the phenomenon that the strain begins to localize along with load increasing has been found, and the strain distributions are different considering different meshes even under the same loads.

It is well-known, the incorporation of damage into a finite element analysis without special technique [28-38] results in pathologically mesh sensitivity. This occurs because, as the mesh is refined, there is a tendency for the damage zone to localize to a zero volume. On the other hand, from the viewpoint of

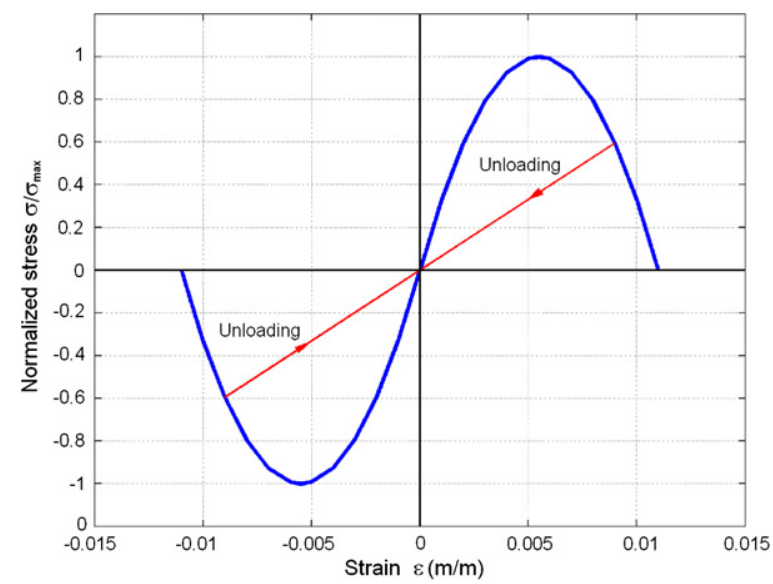

Fig. 4. Stress vs. strain $(\sigma-\varepsilon)$ curve of the orientational component.

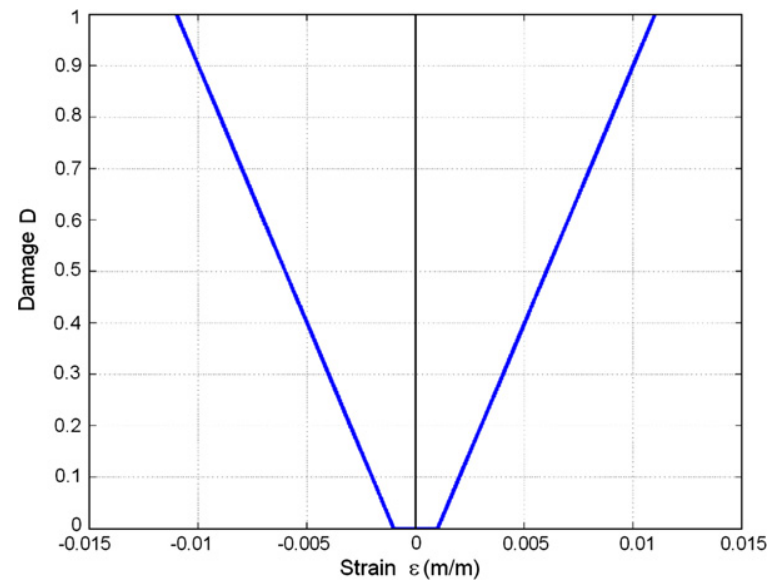

Fig. 5. Damage vs. strain $(D-\varepsilon)$ curve of the orientational component. 


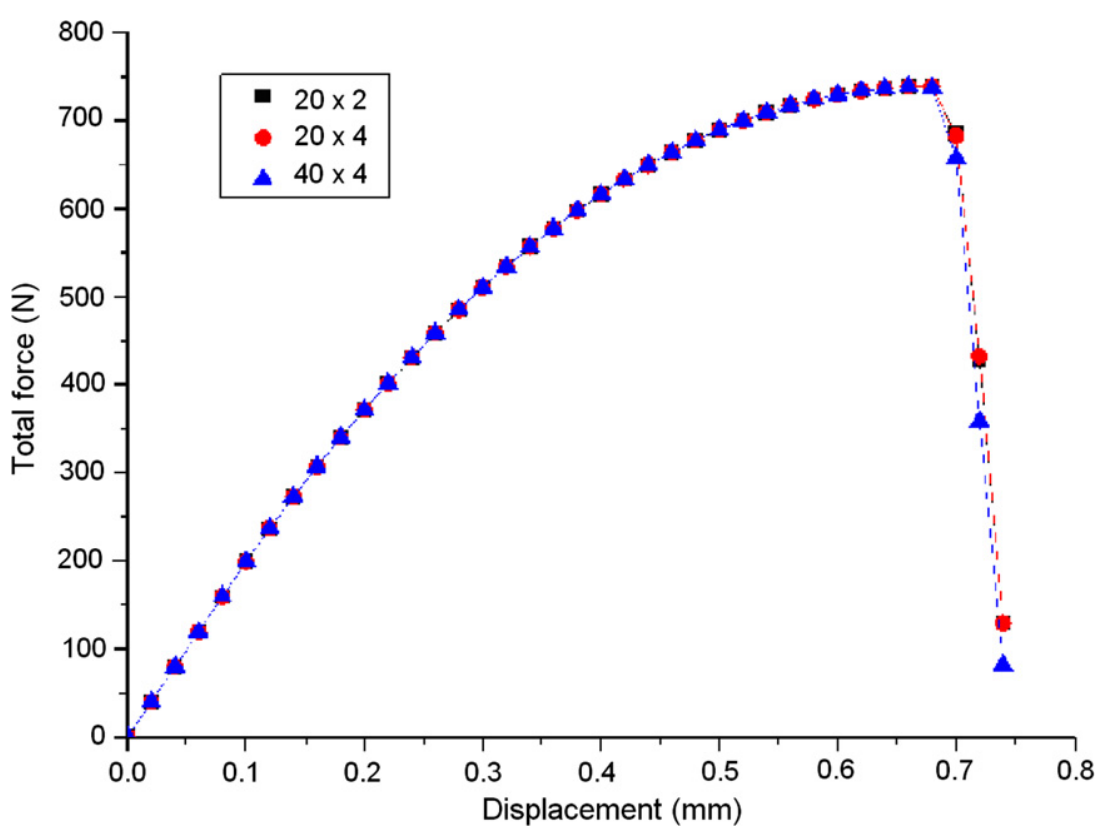

Fig. 6. Two-dimensional plate with imperfection - load-deflection curves.
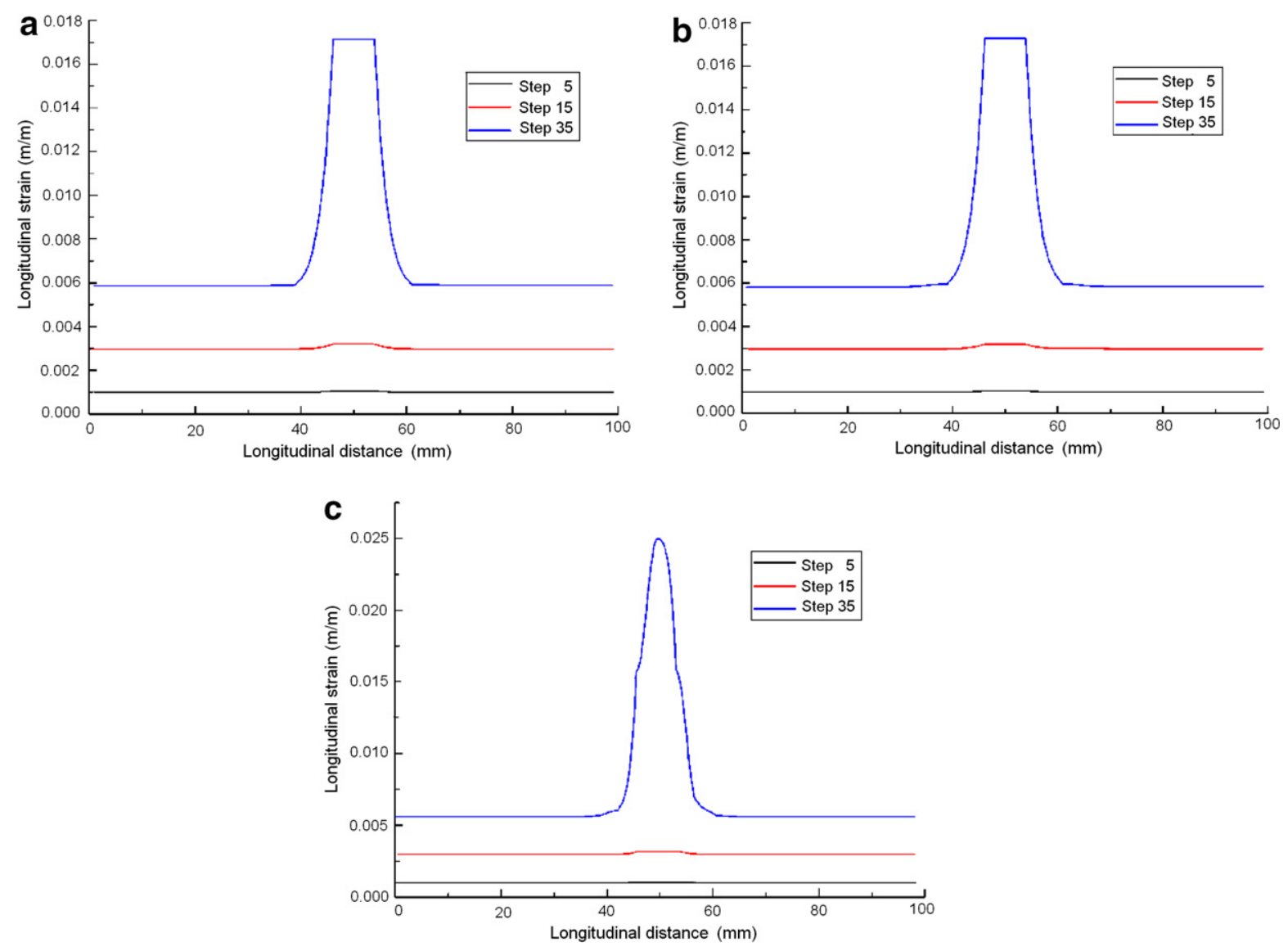

Fig. 7. Evolution of longitudinal strain distribution in plate with imperfection (a, $20 \times 2 ; \mathrm{b}, 20 \times 4 ; \mathrm{c}, 40 \times 4)$. 
mathematics, damage changes the type of governing equations, e.g. elliptic equations to hyperbolic ones in statics and reverse in dynamics [29-33]. Mesh sensitivity leads to the prediction of structural failure with zero energy dissipation, which is physically impossible. For overcoming these difficulties, a simple but powerful method is by incorporating implicit strain gradient [29-33]. Eq. (25) is modified as

$$
D=D\left(\bar{\lambda}_{h}\right)
$$

where $\bar{\lambda}_{h}$ denotes the non-local component's strain history, and it correlates with the local strain history $\lambda_{h}$ by

$$
\bar{\lambda}_{h}-c \Delta \bar{\lambda}_{h}=\lambda_{h}
$$

the internal length scale is preserved in the gradient coefficient $c$, which is of the dimension length squared. $\Delta$ denotes the Laplacian operator.

As shown in Figs. 6 and 7, for given imperfection, as this example, its load-deflecting curve changes little and the width of strain localization is same for different mesh sizes. The proposed model works like non-local $[32,33]$ or strain gradient ones [29,31-35], it can be interpreted as follows. In component assembling model, damage is defined on component but not representative material element (RVE), that is to say, even one or more components damage but the others do not, according to Eq. (27), its governing equation keeps its type unchanged. On the contrary, due to damage and its evolution defined on RVE, the governing equations of classical continuum damage models are prone to change their types and lead to mesh sensitivity. The component assembling model helps to numerical robustness.

\section{Conclusions}

A new material model named component assembling one has been proposed based on pair-functional theory. In which, two kinds of components - the orientational and volumetric ones have been derived from pair potentials and embedding energy respectively. As a typical 1-D component, the orientational one can bear tensile or compressive load. But, the volumetric component is a typical 3-D component and it can bear hydrostatic-pressure. Differing with continuum mechanics, the basic researched element in the proposed model is not RVE but two kinds of components. Moreover, these components are re-division of RVE according to different physical mechanisms. Material has been treated as a component assembly, and its constitutive equations have been formed by assembling these all components' response functions.

The proposed model has been developed for quasi-brittle damage. In which, anisotropy and its evolution have been incorporated appropriately via the component concept. Theoretical analysis and numerical computing indicate that the proposed model has the capacity of re-expressing the generalized Hooke's law and reproducing some results satisfactorily, with the advantages of great conceptual simplicity, physical explicitness, and intrinsic induced anisotropy etc. In addition, the proposed model has the capacity of eliminating mesh sensitivity just like non-local and implicit strain gradient models do.

\section{Acknowledgement}

This research work was sponsored by National Natural Science Foundation of China through Grant No. 10572140 and No. 10232050, and also partly by Ministry of Science and Technology Foundation No. 2002 CB412706.

\section{References}

[1] D. Raabe, Computational Material Science - The Simulation of Materials Microstructures and Properties, Wiley-VCH, 1998.

[2] D. Raabe, F. Roters, F. Barlet, L.Q. Chen, Continuum Scale Simulation of Engineering Materials: Fundamentals-MicrostructuresProcess Applications, Wiley-VCH Verlag GmbH \& Co. KGaA, Weinheim, 2004.

[3] R. Phillips, Crystals, Defects and Microstructures-Modeling Across Scales, Cambridge University Press, 2001.

[4] L.D. Landau, E.M. Lifshits, Quantum Mechanics: Non-relativistic Theory, Pergamon Press, 1977.

[5] M. Born, K. Huang, Dynamical Theory of Crystal Lattices, Clarendon Press, 1954.

[6] M.S. Daw, M.I. Baskes, Embedded-atom-method: derivation and application to impurities, surfaces, and other defects in metals, Phys. Rev. B 29 (1984) 6443-6453. 
[7] M.S. Daw, M.I. Baskes, C.L. Bisson, W.G. Wolfer, Application of the embedded atom method to fracture, dislocation dynamics and hydrogen embrittlement, in: R.H. Jones, W.W. Gerberich (Eds.), Modeling Environmental Effects on Crack Growth Processes, 1986, pp. 99-124.

[8] S.M. Foiles, M.I. Baskes, M.S. Daw, Embedded-atom-method functions for the fcc metals Cu, Ag, Ni, Pt and their alloys, Phys. Rev. B 33 (1986) 7983-7991.

[9] K. Machida, Principles of Molecular Mechanics, Wiley, 1999.

[10] K. Ohno, K. Esfarjani, Y. Kawazoe, Computational Materials Science - From Ab Initio to Monte Carlo Methods, Springer, 1999.

[11] X. Leech, R. Andrew, Molecular Modeling, Principles and Applications, second ed., Prentice Hall, 2001.

[12] Y.C. Fung, Foundations of Solid Mechanics, Prentice Hall, Englewood Cliffs, NJ, 1965.

[13] R. Hill, The Mathematical Theory of Plasticity, Oxford, 1950.

[14] L.M. Kachanov, On the time to failure under creep condition, Izv. Akad. Nauk. USSR. Otd. Tekhn. Nauk 8 (1958) $26-31$.

[15] L.M. Kachanov, Introduction to Continuum Damage Mechanics, Martinus Nijhoff Publishers, 1986.

[16] J. Lemaitre, A Course on Damage Mehanics, Springer-Verlag, 1992.

[17] J. Lemaitre, R. Desmorat, Engineering Damage Mechanics: Ductile, Creep, Fatigue and Brittle Failures, Springer, 2005.

[18] D. Krajcinovic, Damage Mechanics, Elsevier, Amsterdam, New York, 1996.

[19] R. Miller, E.B. Tadmor, R. Phillips, M. Ortiz, Quasicontinuum simulation of fracture at the atomic scale, Model. Simul. Mater. Sci. Eng. 6 (1998) 607-638.

[20] R. Miller, M. Ortiz, R. Phillips, V. Shenoy, E.B. Tadmor, Quasicontinuum models of fracture and plasticity, Eng. Fract. Mech. 61 (1998) 427-444.

[21] H.J. Gao, P. Klein, Numerical simulation of crack growth in an isotropic solid with randomized internal cohesive bonds, J. Mech. Phys. Solids 46 (2) (1998) 187-218.

[22] Y.P. Chen, D.L. James, Connecting molecular dynamics to microporphic theory (I, II), Physica A 322 (2003) $359-392$.

[23] Y.P. Chen, D.L. James, A. Eskandarian, Atomistic viewpoint of the applicability of microcontinuum theories, Int. J. Solid Struct. 41 (2004) 2085-2097.

[24] J. Lemaitre, Local approach of fracture, Eng. Fract. Mech. 25 (5/6) (1986) 523-537.

[25] J.L. Chaboche, Continuum damage mechanics: Part I general concepts, and Part II damage growth, crack initiation, and crack growth, J. Appl. Mech. 55 (1988) 59-72.

[26] Y.N. Rabotnov, Creep rupture, in: M. Hetenyi, W.G. Vincenti (Eds.), Applied Mechanics, Proceedings of the Twelfth International Congress of Applied Mechanics, Standford-Springer-Verlag, Berlin, 1969, pp. 342-349.

[27] J. Lemaitre, J.L. Chaboche, Mechanics of Solid Materials, Cambridge University Press, 1990 (Translated by Shrivastava, B.).

[28] T. Belytschko, D. Organ, C. Gerlach, Element-free Galerkin methods for dynamic fracture in concrete, Comput. Meth. Appl. Mech. Eng. 187 (2000) 385-399.

[29] J.S. Chen, X.W. Zhang, T. Belytschko, An implicit gradient model by a reproducing kernel strain regularization in strain localization problems, Comput. Meth. Appl. Mech. Eng. 193 (2004) 2827-2844.

[30] A. Needleman, Material rate dependence and mesh sensitivity in localization problems, Comput. Meth. Appl. Meh. Eng. 67 (1988) $69-85$.

[31] R.H.J. Peerlings, R.D. Borst, W.A.M. Brekelmans, J.H.P.D. Vree, Grading enhanced damage for quasi-brittle materials, Int. J. Numer. Meth. Eng. 39 (1996) 3391-3403.

[32] R.H.J. Peerlings, M.G.D. Geers, R.D. Borst, W.A.M. Brekelmans, A critical comparison of nonlocal and gradient-enhanced softening continua, Int. J. Solids Struct. 38 (2001) 7723-7746.

[33] R.H.J. Peerlings, R.D. Borst, W.A.M. Brekelmans, M.G.D. Geers, Localization issues in local and nonlocal continuum approaches to fracture, Eur. J. Mech. A/Solids 21 (2002) 75-189.

[34] E. Kuhl, E. Ramm, R.D. Borst, An anisotropic gradient damage model for quasi-brittle materials, Comput. Meth. Appl. Mech. Eng. 183 (2000) 87-103.

[35] C.S. Chang, H. Askes, L.J. Sluys, Higher-order strain/higher-order stress gradient models derived from a discrete microstructure, with application to fracture, Eng. Fract. Mech. 69 (2002) 1907-1924.

[36] J. Oliver, A.E. Huespe, M.D.G. Pulido, E. Chaves, From continuum mechanics to fracture mechanics: the strong discontinuity approach, Eng. Fract. Mech. 69 (2002) 113-136.

[37] J. Planas, M. Elices, G.V. Guinea, F.J. Gomez, D.A. Cendon, I. Arbilla, Generalizations and specializations of cohesive crack models, Eng. Fract. Mech. 70 (1999) 1759-1776.

[38] Z.J. Yang, J.F. Chen, Fully automatic modeling of cohesive discrete crack propagation in concrete beams using local arc-length methods, Int. J. Solids Struct. 41 (3-4) (2004) 801-826. 